\title{
Controller Design and Analysis of Spacecraft Automatic Levelling and Equalizing Hoist Device based on Hanging Point Adjustment
}

\author{
Tang Laiying ${ }^{1,2}$, Liu Guangtong ${ }^{1,2}$, Guo Tao ${ }^{1,2}$, Liu Zhe ${ }^{1,2}$, Hu Ruiqin ${ }^{1,2}$, Guo Dabao ${ }^{1,2}$ \\ ${ }^{1}$ Beijing Institute of Spacecraft Environment Engineering, 100094 Beijing China \\ ${ }^{2}$ Beijing Engineering Research Center of the Intelligent Assembly Technology and Equipment for Aerospace Product, 100094 Beijing China
}

\begin{abstract}
Spacecraft Automatic Levelling and Equalizing Hoist Device (SALEHD) is a kind of hoisting device developed for eccentric spacecraft level-adjusting, based on hanging point adjustment by utilizing XY-workbench. To make the device automatically adjust the spacecraft to be levelling, the controller for SALEHD was designed in this paper. Through geometry and mechanics analysis for SALEHD and the spacecraft, the mathematical model of the controller is established. And then, the link of adaptive control and the link of variable structure control were added into the controller to adapt the unknown parameter and eliminate the interference of support vehicle. The stability of the controller was analysed, through constructing Lyapunov energy function. It was proved that the controller system is asymptotically stable, and converged to origin that is equilibrium point. So the controller can be applied in SALEHD availably and safely.
\end{abstract}

\section{Introduction}

Hoist device, as an important actuating unit in spacecraft hoisting, has been widely used in assembly and integration of spacecraft, such as propellant tank assembling, modules docking, spacecraft environment test, containing and transportation for spacecraft. So hoisting is an important process for quality control and safety guarantee in spacecraft assembly ${ }^{[1][2]}$.

However, in spacecraft hoisting currently, the deviation of spacecraft centre of gravity (COG) from its geometric centre or theoretical centre is contributed to differences in assembly degree and operating condition. This would become hidden safety trouble such as inclinations, swing and clash, by special hoisting device of traditional fixed structure which could not self-adapt the eccentric spacecraft. So, self-adapted electromechanical hoist device needs to be researched to resolve these problems ${ }^{[3][4][5]}$.

In this paper, adaptive controller based on hanging point adjustment by utilizing XY-workbench was designed, and stability of the controller was analysed, which is proved to be asymptotically stable and converged to equilibrium point.

\section{Brief introduction of SALEHD}

\subsection{Composition of SALEHD ${ }^{[1][2]}$}

SALEHD based on hanging point adjustment, mainly consists of mechanical part and electric part. Mechanical part consists of hanging beam, $\mathrm{XY}$-workbench, ring and so on. Electric part consists of tilt sensor, tension sensors, electrical cabinet, LED displays, motors, power supply and control software. Compositions of SALEHD are shown in Figure 1.

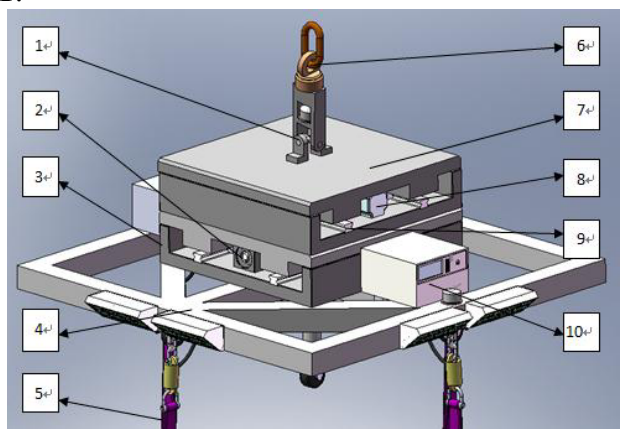

1-Universal joint; 2-ball screw; 3-Y sliding platform; 4hanging beam; 5-sling; 6-ring; 7-X sliding platform; 8motor; 9-slide; 10-electrical cabinet

Figure 1. Internal structure diagram of SALEHD

\subsection{Workflow of SALEHD ${ }^{[1][2]}$}

The specific level-adjusted workflow is described as follows:

(1) Spacecraft and SALEHD is connected by slings under SALEHD. Slings are adjusted to equal length, and preloaded.

(2) Point moving the crane to hoist spacecraft a little height, makes spacecraft generate a small inclination angle, and docking frame of spacecraft is suspended partially from docking frame of support vehicle. 
(3) Horizontal degree of docking frame of spacecraft is measured by two-dimensional tilt sensor, after spacecraft is static.

(4) Spacecraft is landed back on the support vehicle.

(5) XY-workbench is moved to the specified location which is calculated by controller through the sensors.

(6) Repeat steps above, until the horizontal degree is within the allowable range.

\section{Establishment of mathematical model}

\subsection{Simplification of boundary conditions ${ }^{[1][2]}$}

(1) Spacecraft and support vehicle are in a horizontal state, before hoisting.

(2) Slings between spacecraft and SALEHD are equal length. And stress deformations of slings are ignored.

(3) Spacecraft and SALEHD are regarded as rigid bodies, whose stress deformations are ignored.

(4) Attitude angle between spacecraft and SALEHD are regarded as equal in static state, because slings are vertical and their lengths are equal.

\subsection{Model building}

According the simplification above, the system consisted by spacecraft and SALEHD is shown as Figure. 2.
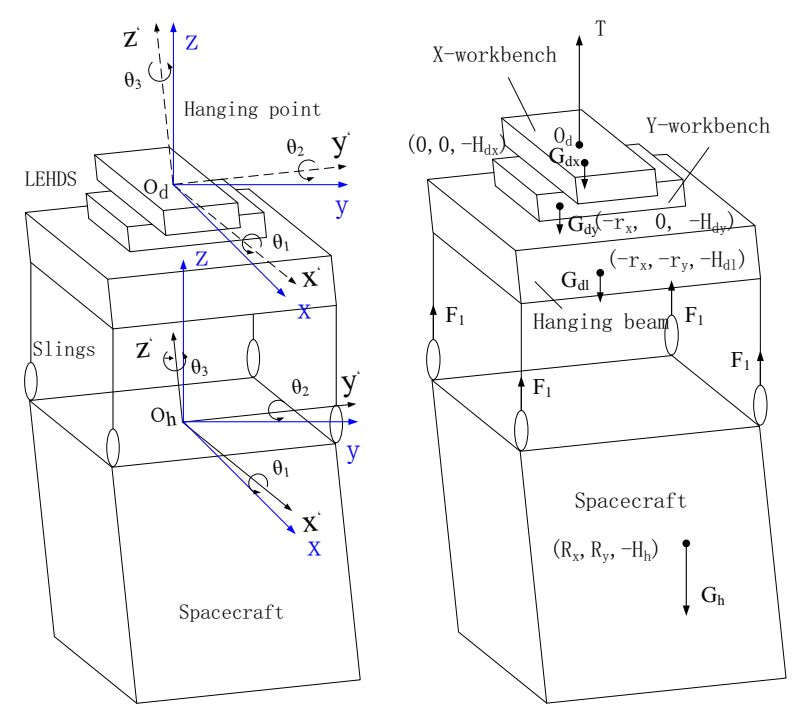

Figure 2. Schematic diagram of inclined system

\subsubsection{Geometric model building}

Taking hanging point $O_{d}$ as the origin, body coordinate $\Sigma O_{d} X_{d}{ }^{\prime} Y_{d}{ }^{\prime} Z_{d}$ ' is established. So, position vectors of COGs of $\mathrm{X}$-workbench, $\mathrm{Y}$-workbench and hanging beam can be expressed as Formula (1).

Where

$$
\left\{\begin{array}{l}
\vec{V}_{G d x}^{\prime}=\left(0,0,-H_{d x}\right)^{T} \\
\vec{V}_{G d y}^{\prime}=\left(-r_{x}, 0,-H_{d y}\right)^{T} \\
\vec{V}_{G d l}^{\prime}=\left(-r_{x},-r_{y},-H_{d l}\right)^{T}
\end{array}\right.
$$

$H_{d x}, H_{d y}, H_{d l}$-respectively as distance from COGs of Xworkbench, $\mathrm{Y}$-workbench and hanging beam, to hanging point above SALEHD, m;

$r_{x}, r_{y}$-respectively as displacement of X-workbench and Y-workbench, m;

Taking spacecraft surface centre $O_{h}$ as the origin, body coordinate $\Sigma O_{h} X_{h}{ }^{\prime} Y_{h}{ }^{\prime} Z_{h}$ ' is established. So, position vectors of COGs of spacecraft can be expressed as Formula (2).

$$
\vec{V}_{G h}^{\prime}=\left(R_{x}, R_{y},-H_{h}\right)^{T}
$$

Where

$R_{x}, R_{y},-H_{d}$-respectively as position of COG of spacecraft, $\mathrm{m}$;

\subsubsection{Mechanical model building}

1) Attitude and coordinate transformation for SALEHD

When SALEHD is tilted, tilted angle is less than $1^{\circ}$. So Rotation matrix could be simplified as Formula (3), approximated by infinitesimal angular displacement.

$$
C_{d}=E-\boldsymbol{\Phi}^{\times}=\left(\begin{array}{ccc}
1 & -\theta_{3} & \theta_{2} \\
-\theta_{3} & 1 & -\theta_{1} \\
\theta_{2} & -\theta_{1} & 1
\end{array}\right)=\left(\begin{array}{ccc}
1 & 0 & \theta_{2} \\
0 & 1 & -\theta_{1} \\
-\theta_{2} & \theta_{1} & 1
\end{array}\right)
$$

Where

$\theta_{1}, \theta_{2}, \theta_{3}$-respectively as turning angles around the axis $\mathrm{X}$, $\mathrm{Y}$, and $\mathrm{Z}$ of body coordinate of SALEHD. (Because of no moment in yaw direction, $\theta_{3}=0$ )

SALEHD can be regarded as rotating around the hanging point above, so taking hanging point $O_{d}$ as the origin, inertia coordinate $\Sigma O_{d} X Y Z$ is established. COG coordinate value of each part of SALEHD can be expressed as Formula (4).

$$
\left\{\begin{array}{l}
\vec{V}_{G d x}=C_{d} \vec{V}_{G d x}^{\prime}=\left[-\theta_{2} H_{d x}, H_{d x} \theta_{1},-H_{d x}\right]^{T} \\
\vec{V}_{G d y}=C_{d} \vec{V}_{G d y}^{\prime}=\left[-r_{x}-\theta_{2} H_{d y}, \theta_{1} H_{d y}, \theta_{2} r_{x}-H_{d y}\right]^{T} \\
\vec{V}_{G d l}=C_{d} \vec{V}_{G d l}^{\prime}=\left[-r_{x}-\theta_{2} H_{d l},-r_{y}+\theta_{1} H_{d l}, \theta_{2} r_{x}-\theta_{1} r_{y}-H_{d l}\right]^{T}
\end{array}\right.
$$

2) Attitude and coordinate transformation for spacecraft.

When spacecraft is hoisted in static state, each sling is in vertical state, so COG coordinate value of spacecraft can be expressed in inertia coordinate $\Sigma O_{d} X Y Z$ as Formula (5)

$$
\vec{V}_{G h}=C\left(\vec{V}_{G h}^{\prime}-\vec{V}_{h d 1}^{\prime}\right)+(0,0,-L)^{T}+C \vec{V}_{d d 1}^{\prime}
$$

Where

$L$ - as length of sling between spacecraft and SALEHD, m;

Formula (1), (2), (3), (4) are taken into (5) to obtain (6)

$$
\vec{V}_{G h}=\left[\begin{array}{l}
-r_{x}-\theta_{2} H_{d j}+R_{x}-\theta_{2} H_{h} \\
-r_{y}+\theta_{1} H_{d j}+R_{y}+\theta_{1} H_{h} \\
\theta_{2} r_{x}+\theta_{1} r_{y}-H_{d j}-L-\theta_{2} R_{x}+\theta_{1} R_{y}-H_{h}
\end{array}\right]
$$

Where

$H_{d j}$-as vertical distance between hanging point below and above of SALEHD, m;

3) Moment balance

The force balance equation is established for the spacecraft as Formula (7).

$$
G_{h}=F_{1}+F_{2}+F_{3}+F_{4}
$$

The static torque balance equation is established for spacecraft in origin $O_{d}$ of inertia coordinate $\Sigma O_{d} X Y Z$. 
$\sum M=\vec{V}_{G d x} \times\left[\begin{array}{l}0 \\ 0 \\ -G_{d x}\end{array}\right]+\vec{V}_{G d x} \times\left[\begin{array}{l}0 \\ 0 \\ -G_{d y}\end{array}\right]+\vec{V}_{G d l} \times\left[\begin{array}{l}0 \\ 0 \\ -G_{d l}\end{array}\right]+\vec{V}_{G h} \times\left[\begin{array}{l}0 \\ 0 \\ -G_{h}\end{array}\right]=0$

Formula (1), (7) are taken into (8) to obtain (9)

$$
\left\{\begin{array}{l}
\theta_{1}=\frac{r_{y} G_{d l}+r_{y} G_{h}-R_{y} G_{h}}{H_{d x} G_{d x}+H_{d y} G_{d y}+H_{d l} G_{d l}+H_{d j} G_{h}+H_{h} G_{h}} \\
\theta_{2}=\frac{R_{x} G_{h}-r_{x} G_{d y}-r_{x} G_{d l}-r_{x} G_{h}}{H_{d x} G_{d x}+H_{d y} G_{d y}+H_{d l} G_{d l}+H_{d j} G_{h}+H_{h} G_{h}}
\end{array}\right.
$$

When SALEHD is levelling, $\theta_{I}=\theta_{2}=0$, i.e.

$$
\left\{\begin{array}{l}
r_{y p}=\frac{R_{y} G_{h}}{G_{d l}+G_{h}} \\
r_{x p}=\frac{R_{x} G_{h}}{G_{d y}+G_{d l}+G_{h}}
\end{array}\right.
$$

Where

$r_{x p}, r_{y p}$-respectively as displacement of X-workbench and Y-workbench when SALEHD is leveling, $\mathrm{m}$;

\section{Design for variable structure adaptive controller}

\subsection{Control in known parameter and total suspended}

From Formula (9) and (10), (11) can be obtained:

$$
\left\{\begin{array}{l}
\Delta r_{y}=r_{y p}-r_{y}=-\frac{H_{d x} G_{d x}+H_{d y} G_{d y}+H_{d l} G_{d l}+H_{d j} G_{h}+H_{h} G_{h}}{G_{d l}+G_{h}} \theta_{1} \\
\Delta r_{x}=r_{x p}-r_{x}=\frac{H_{d x} G_{d x}+H_{d y} G_{d y}+H_{d l} G_{d l}+H_{d j} G_{h}+H_{h} G_{h}}{G_{d y}+G_{d l}+G_{h}} \theta_{2}
\end{array}\right.
$$

Where

$\Delta r_{x}, \Delta r_{y p}$ - respectively as difference from location to target position of X-workbench and $\mathrm{Y}$-workbench, $\mathrm{m}$;

In the condition of known parameter of spacecraft and SALEHD (such as $G_{h}$ ), $\Delta r_{x}, \Delta r_{y p}$ can be calculated from Formula (11) and the tilt angles $\theta_{1}, \theta_{2}$. So motor can drive $\mathrm{XY}$-workbench to equilibrium point by $\Delta r_{x p}, \Delta r_{y p}$, to make spacecraft levelling. System block diagram is shown in Figure.3.

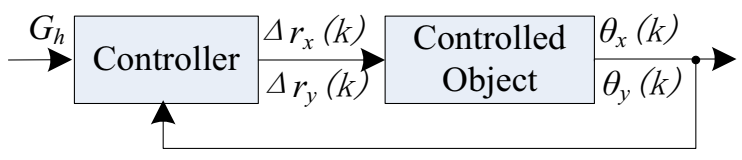

Figure 3. System block diagram

\subsection{Adaptive control in unknown parameter and total suspended}

In actual hoisting process, $G_{h}, H_{h}, R_{x}, R_{y}$ are unknown, so they need to be estimated and identified. $G_{h}$ can be approximated by tension sum of slings through Formula (7). $R_{x}, R_{y}$ can be calculated by equilibrium position of XYworkbench through Formula (10). Only $H_{h}$ can't be calculated and its influences decrease with the tilt angle decreasing, so it needs to be predicted, identified and corrected. Parameter identification module is added into control system, shown in Figure 4.

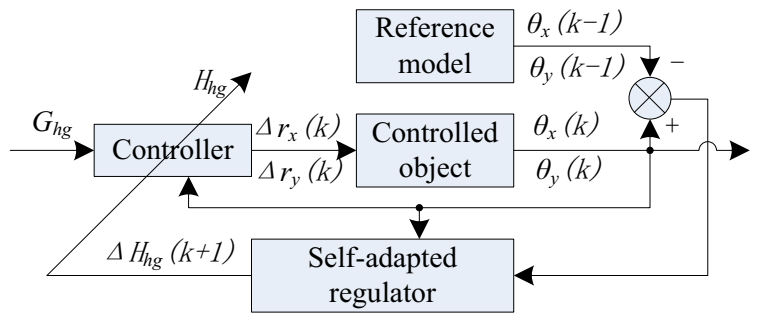

Figure 4. Block diagram of adaptive control system Formula (7) and (11) are discretized as follows:

1) Discrete equation of controller

$$
\left\{\begin{array}{c}
\Delta r_{x}(k+1)=\frac{H_{d x} G_{d x}+H_{d y} G_{d y}+H_{d l} G_{d l}+H_{d j} G_{h g}+G_{h g} H_{h g}(k+1)}{G_{d y}+G_{d l}+G_{h g}} \theta_{2}(k) \\
\Delta r_{y}(k+1)=-\frac{H_{d x} G_{d x}+H_{d y} G_{d y}+H_{d l} G_{d l}+H_{d j} G_{h g}+G_{h g} H_{h g}(k+1)}{G_{d l}+G_{h g}} \theta_{1}(k) \\
G_{h g}=F_{1}+F_{2}+F_{3}+F_{4}
\end{array}\right.
$$

2) Discrete equation of controlled object

$$
\left\{\begin{array}{c}
\theta_{1}(k+1)=\frac{\left(G_{d l}+G_{h}\right) \sum_{i=0}^{k+1} \Delta r_{y}(i)-R_{y} G_{h}}{H_{d x} G_{d x}+H_{d y} G_{d y}+H_{d l} G_{d l}+H_{d j} G_{h}+H_{h} G_{h}} \\
\theta_{2}(k+1)=\frac{R_{x} G_{h}-\left(G_{d y}+G_{d l}+G_{h}\right) \sum_{i=0}^{k+1} \Delta r_{x}(i)}{H_{d x} G_{d x}+H_{d y} G_{d y}+H_{d l} G_{d l}+H_{d j} G_{h}+H_{h} G_{h}}
\end{array}\right.
$$

Comparing two output values $\theta$, the relation between $H_{h}$ and $\theta$ can be obtained, as follows:

$\left\{\begin{array}{l}\frac{\theta_{1}(k+1)}{\theta_{1}(k)}=\frac{\left[H_{h}-H_{h g}(k+1)\right] G_{h}}{H_{d x} G_{d x}+H_{d y} G_{d y}+H_{d l} G_{d l}+H_{d j} G_{h}+H_{h} G_{h}} \\ \frac{\theta_{2}(k+1)}{\theta_{2}(k)}=\frac{\left[H_{h}-H_{h g}(k+1)\right] G_{h}}{H_{d x} G_{d x}+H_{d y} G_{d y}+H_{d l} G_{d l}+H_{d j} G_{h}+H_{h} G_{h}}\end{array}\right.$

Discrete state equation of parameter regulator is obtained, through corrected value $H_{h g}(k+2)$ replacing true value $H_{h}$.

$$
\left\{\begin{aligned}
H_{h g}(k+2)= & \frac{H_{d x} G_{d x}+H_{d y} G_{d y}+H_{d l} G_{d l}+H_{d j} G_{h}+G_{h} H_{h g}(k+1)}{G_{h}\left[\theta_{1}(k)-\theta_{1}(k+1)\right]} \theta_{1}(k+1) \\
& +H_{h g}(k+1) \\
H_{h g}(k+2)= & \frac{H_{d x} G_{d x}+H_{d y} G_{d y}+H_{d l} G_{d l}+H_{d j} G_{h}+G_{h} H_{h g}(k+1)}{G_{h}\left[\theta_{2}(k)-\theta_{2}(k+1)\right]} \theta_{2}(k+1) \\
& +H_{h g}(k+1)
\end{aligned}\right.
$$

And reference model is the K-1 time response model of the system, so discrete state equation of reference model can be described as follows:

$$
\left\{\begin{array}{l}
\theta_{1}(k+1)=\theta_{1}(k) \\
\theta_{2}(k+1)=\theta_{2}(k)
\end{array}\right.
$$

\subsection{Variable structure adaptive control in unknown parameter and support vehicle}

Before spacecraft leaving support vehicle, support vehicle makes a restrictive effect to prevent spacecraft from inclining further, compared with the total suspended state. The output value tends to a constant value, showing a certain saturation, which makes structure of the control system changed. So the influence of the support vehicle can be equivalent to a saturation nonlinearity series in output port of adaptive control system in unknown parameter and total suspended, as follows: 


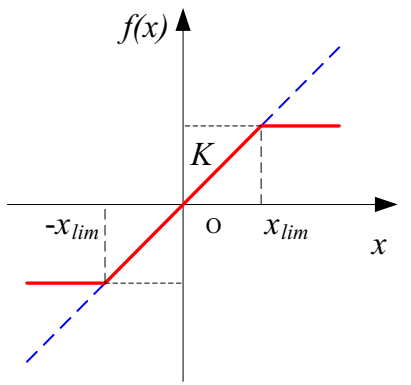

Figure 5. Chart of saturation nonlinearity

$$
f(x)=\left\{\begin{array}{l}
K x ;|x| \leq x_{\lim } \\
K x_{\lim } ; x>x_{\lim } \quad K=1, x_{\lim }>0 \\
-K x_{\lim } ; x<-x_{\lim }
\end{array}\right.
$$

The system works in two different ranges. When the system working in the range of linearity, control mode is the adaptive control in total suspended. When the system working in the range of saturation nonlinearity, the output value tends to a constant value leading to the difference between reference model and actual output value is zero, which make the self-adapted system can't identify parameter or adjust parameter. So a judgment link is added in front of the self-adapted regulator, as follows.

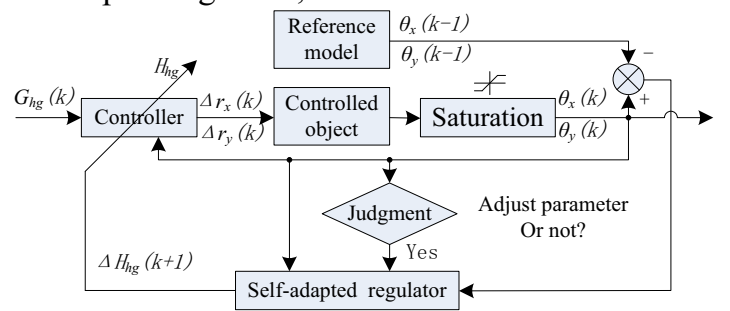

Figure 6. Block diagram of variable structure adaptive

$$
\begin{aligned}
& \text { control system } \\
& H_{h g}(k+2)=\left\{\begin{array}{l}
H_{h g}(k+1) ;\left|\theta_{1}(k)\right| \geq \theta_{\lim }-\delta, \text { 且 }\left|\theta_{2}(k)\right| \geq \theta_{\lim }-\delta \\
\frac{\theta_{1}(k+1)}{\theta_{1}(k)-\theta_{1}(k+1)} K+H_{h g}(k+1) ;\left|\theta_{1}(k)\right|<\theta_{\lim }-\delta \\
\frac{\theta_{2}(k+1)}{\theta_{2}(k)-\theta_{2}(k+1)} K+H_{h g}(k+1) ;\left|\theta_{2}(k)\right|<\theta_{\lim }-\delta
\end{array}\right. \\
& K=\frac{H_{d x} G_{d x}+H_{d y} G_{d y}+H_{d l} G_{d l}+H_{d j} G_{h}(k+1)+G_{h}(k+1) H_{h g}(k+1)}{G_{h}(k+1)}(18)
\end{aligned}
$$

Let: $x_{1}=\Delta r_{x} ; x_{2}=\Delta r_{y} ; \quad x_{3}=\theta_{1} ; \quad x_{4}=\theta_{2} ; \quad r=G_{h g} ;$

The final state equation of whole control system as follows:

$$
\begin{aligned}
& x_{1}(k+1)=\frac{H_{d x} G_{d x}+H_{d y} G_{d y}+H_{d l} G_{d l}+H_{d j} r(k+1)+r(k+1) H_{h g}(k+1)}{G_{d y}+G_{d l}+r(k+1)} x_{4}(k) \\
& x_{2}(k+1)=-\frac{H_{d x} G_{d x}+H_{d y} G_{d y}+H_{d l} G_{d l}+H_{d j} r(k+1)+r(k+1) H_{h g}(k+1)}{G_{d l}+r(k+1)} x_{3}(k) \\
& \left\{x_{3}(k+1)=f\left(\frac{\left(G_{d l}+G_{h}\right) \sum_{i=0}^{k+1} x_{2}(i)-R_{y} G_{h}}{H_{d x} G_{d x}+H_{d y} G_{d y}+H_{d l} G_{d l}+H_{d j} G_{h}+H_{h} G_{h}}\right)\right. \\
& x_{4}(k+1)=f\left(\frac{R_{x} G_{h}-\left(G_{d y}+G_{d l}+G_{h}\right) \sum_{i=0}^{k+1} x_{1}(i)}{H_{d x} G_{d x}+H_{d y} G_{d y}+H_{d l} G_{d l}+H_{d j} G_{h}+H_{h} G_{h}}\right) \\
& r(k)=F_{1}(k)+F_{2}(k)+F_{3}(k)+F_{4}(k) \\
& \left\{\begin{array}{l}
y_{1}(k+1)=x_{3}(k+1) \\
y_{2}(k+1)=x_{4}(k+1)
\end{array}\right.
\end{aligned}
$$

Where

$$
\begin{gathered}
f(x)=\left\{\begin{array}{l}
x ;|x| \leq x_{\lim } \\
x_{\lim } ; x>x_{\lim } \\
-x_{\lim } ; x<-x_{\lim }
\end{array}\right. \\
H_{h g}(k+1)=\left\{\begin{array}{l}
H_{h g}(k) ;\left|x_{3}(k-1)\right| \geq x_{\lim }, \text { 且 }\left|x_{4}(k-1)\right| \geq x_{\lim } \\
\frac{x_{3}(k)}{x_{3}(k-1)-x_{3}(k)} K(k)+H_{h g}(k) ;\left|x_{3}(k-1)\right|<x_{\lim } \\
\frac{x_{4}(k)}{x_{4}(k-1)-x_{4}(k)} K(k)+H_{h g}(k) ;\left|x_{4}(k-1)\right|<x_{\lim }
\end{array}\right. \\
K(k)=\frac{H_{d x} G_{d x}+H_{d y} G_{d y}+H_{d l} G_{d l}+H_{d j} r(k)+r(k) H_{h g}(k)}{r(k)}
\end{gathered}
$$

\section{Stability analyses}

\subsection{Stability analysis for Adaptive control in total suspended}

Adaptive control system is nonlinear essentially, so the Lyapunov stability theorem is adopted to judge stability for the system. Positive definite quadratic function is introduced as Lyapunov energy function $V[x(k)]$ for discrete system, i.e.

$$
V[\boldsymbol{x}(k)]=\boldsymbol{x}^{T}(k) P \boldsymbol{x}(k)
$$

Where

$$
\begin{gathered}
\boldsymbol{x}(k)=\left[x_{1}(k), x_{2}(k), x_{3}(k), x_{4}(k)\right]^{T} \\
P=\left[\begin{array}{cccc}
1 & 0 & 0 & 0 \\
0 & 1 & 0 & 0 \\
0 & 0 & 1 & 0 \\
0 & 0 & 0 & 1
\end{array}\right]
\end{gathered}
$$

i.e.

$$
V[\boldsymbol{x}(k)]=x_{1}^{2}(k)+x_{2}^{2}(k)+x_{3}^{2}(k)+x_{4}^{2}(k)
$$

$V[x(k)]$ is positive definite, and the origin is balanced. If $\Delta V[x(k)]$ is negative definite, the system is asymptotically stable. i.e.

$$
\begin{aligned}
\Delta V[\boldsymbol{x}(k)] & =V[\boldsymbol{x}(k+1)]-V[\boldsymbol{x}(k)] \\
= & {\left[x_{1}^{2}(k+1)-x_{1}^{2}(k)\right]+\left[x_{2}^{2}(k+1)-x_{2}^{2}(k)\right] } \\
+ & {\left[x_{3}^{2}(k+1)-x_{3}^{2}(k)\right]+\left[x_{4}^{2}(k+1)-x_{4}^{2}(k)\right] }
\end{aligned}
$$

Because the absolute value of the differences between true value and estimated value of COG height is far less than the true value. i.e.

$$
\left|H_{h}-H_{h g}(k)\right|<\left|H_{h}\right|
$$

Formula (22) is taken into (14) to obtain as follows

$$
\left\{\begin{aligned}
\left|\frac{x_{3}(k+1)}{x_{3}(k)}\right|= & \left|\frac{\left[H_{h}-H_{h g}(k)\right] G_{h}}{H_{d x} G_{d x}+H_{d y} G_{d y}+H_{d l} G_{d l}+H_{d j} G_{h}+H_{h} G_{h}}\right| \\
& <\left|\frac{\left[H_{h}-H_{h g}(k)\right] G_{h}}{H_{h} G_{h}}\right|<1 \\
\left|\frac{x_{4}(k+1)}{x_{4}(k)}\right|= & \left|\frac{\left[H_{h}-H_{h g}(k)\right] G_{h}}{H_{d x} G_{d x}+H_{d y} G_{d y}+H_{d l} G_{d l}+H_{d j} G_{h}+H_{h} G_{h}}\right| \\
& <\left|\frac{\left[H_{h}-H_{h g}(k)\right] G_{h}}{H_{h} G_{h}}\right|<1
\end{aligned}\right.
$$




$$
\left\{\begin{array}{l}
x_{3}^{2}(k+1)-x_{3}^{2}(k)<0 \\
x_{4}^{2}(k+1)-x_{4}^{2}(k)<0
\end{array}\right.
$$

Formula (19) is taken into (23) to obtain as follows

$$
\left\{\begin{array}{l}
\left|\frac{x_{1}(k+1)}{x_{1}(k)}\right|=\left|\frac{x_{4}(k+1)}{x_{4}(k)}\right|<1 \\
\left|\frac{x_{2}(k+1)}{x_{2}(k)}\right|=\left|\frac{x_{3}(k+1)}{x_{3}(k)}\right|<1
\end{array}\right.
$$

i.e.

$$
\left\{\begin{array}{l}
x_{1}^{2}(k+1)-x_{1}^{2}(k)<0 \\
x_{2}^{2}(k+1)-x_{2}^{2}(k)<0
\end{array}\right.
$$

Formula (24) and (26) are taken into (21) to obtain as follows.

$$
\Delta V[x(k)]<0
$$

$\Delta V[x(k)]$ is negative definite, so the system is asymptotically stable and converged to the origin, that is the equilibrium point.

\subsection{Stability analysis for variable structure adaptive control in support vehicle}

Variable structure adaptive control system works in two different ranges. When system works in range of linearity, the stability of the system has been proved above. The stability of the system working in the range of saturation nonlinearity, only needs to be proved in this section.

Positive definite quadratic function is introduced as Lyapunov energy function $V[L(k)]$ for discrete system, i.e.

$$
V[\boldsymbol{L}(k)]=\boldsymbol{L}^{T}(k) P \boldsymbol{L}(k)
$$

Where

$$
\begin{gathered}
\boldsymbol{L}(k)=\left[L_{x}(k), L_{y}(k)\right]^{T} \\
L_{x}(k)=r_{x p}-r_{x}(k)=r_{x p}-\sum_{i=0}^{k} \Delta r_{x}(k) \\
L_{y}(k)=r_{y p}-r_{y}(k)=r_{x p}-\sum_{i=0}^{k} \Delta r_{x}(k) \\
P=\left(\begin{array}{cc}
1 & 0 \\
0 & 1
\end{array}\right)
\end{gathered}
$$

i.e.

$$
V[\boldsymbol{L}(k)]=L_{x}^{2}(k)+L_{y}^{2}(k)
$$

Where

$L_{x}(k), L_{y}(k)$-respectively as difference from location to equilibrium position of $\mathrm{X}$-workbench or $\mathrm{Y}$-workbench, m; i.e.

$V[L(k)]$ is positive definite, and the origin is balanced. If $\triangle V[L(k)]$ is negative definite, the system is asymptotically stable. i.e.

$$
\begin{aligned}
& \Delta V[\boldsymbol{L}(k)]=V[\boldsymbol{L}(k+1)]-V[\boldsymbol{L}(k)] \\
& =L_{x}^{2}(k+1)+L_{y}^{2}(k+1)-\left[L_{x}^{2}(k)+L_{y}^{2}(k)\right] \\
& =\left[L_{x}^{2}(k+1)-L_{x}^{2}(k)\right]+\left[L_{y}^{2}(k+1)-L_{y}^{2}(k)\right]
\end{aligned}
$$

From Formula (11) and (19), (30) can be obtained as follows:

$$
\begin{aligned}
& L_{x}(k+1)-L_{x}(k)=-\Delta r_{x}(k+1)=-x_{1}(k+1) \\
& =-\frac{H_{d x} G_{d x}+H_{d y} G_{d y}+H_{d l} G_{d l}+H_{d j} r(k+1)+r(k+1) H_{h g}(k+1)}{G_{d y}+G_{d l}+r(k+1)} x_{4}(k) \\
& =-\frac{H_{d x} G_{d x}+H_{d y} G_{d y}+H_{d l} G_{d l}+H_{d j} r(k+1)+r(k+1) H_{h g}(k+1)}{G_{d y}+G_{d l}+r(k+1)} x_{\lim } \\
& \text { Let } \quad x_{\lim }=f\left[x_{4}^{\prime}(k)\right] \\
& R_{x} G_{h}-\left(G_{d y}+G_{d l}+G_{h}\right) \sum_{i=0}^{k+1} x_{1}(i) \\
& x_{4}^{\prime}(k)=\frac{(30)}{H_{d x} G_{d x}+H_{d y} G_{d y}+H_{d l} G_{d l}+H_{d j} G_{h}+H_{h} G_{h}}
\end{aligned}
$$

From Formula (17), it can be obtained that $x_{\text {lim }}$ and $x^{\prime}{ }_{4}(k)$ are both plus or both minus.

Formula (11) and (27) are taken into (31) to obtain as follows

$$
x_{4}^{\prime}(k)=\frac{\left(G_{d y}+G_{d l}+G_{h}\right) L_{x}(k)}{H_{d x} G_{d x}+H_{d y} G_{d y}+H_{d l} G_{d l}+H_{d j} G_{h}+H_{h} G_{h}}
$$

So $x^{\prime}{ }_{4}(k)$ and $L_{x}(k)$ are same plus or same minus.

In summary, it can be obtained that $L_{x}(k+1)-L_{x}(k)$ and $L x(k)$, one is plus and another is minus. Also, the $k$ and $k+1$ time are both in the range of saturation, without crossing zero, so $L_{x}(k+1)$ and $L_{x}(k)$ are both plus or both minus.

If $L_{x}(k+1)>0$ and $L_{x}(k)>0$, then $L_{x}(k+1)-L_{x}(k)<0$, that is $L_{x}(k+1)<L_{x}(k)$, so $\left|L_{x}(k+1)\right|<\left|L_{x}(k)\right|$.

If $L_{x}(k+1)<0$ and $L_{x}(k)<0$, then $L_{x}(k+1)-L_{x}(k)>0$, that is $L_{x}(k+1)>L_{x}(k)$, so $\left|L_{x}(k+1)\right|<\left|L_{x}(k)\right|$.

In summary:

$$
L_{x}^{2}(k+1)-L_{x}^{2}(k)<0
$$

In the same way:

$$
L_{y}^{2}(k+1)-L_{y}^{2}(k)<0
$$

Formula (33) and (34) are taken into (28) to obtain as follows:

$$
\Delta V[\boldsymbol{L}(k)]<0
$$

$\triangle V[L(k)]$ is negative definite, so the system is asymptotically stable and converged to the origin that is the equilibrium point.

In summary, the system working in ranges of linearity and saturation nonlinearity both asymptotically stable, and converged to the same origin.

\section{Conclusion}

The variable structure adaptive controller designed in this paper, can adjust control structure by itself according to the actual support of the vehicle, and adapt different eccentric conditions by correcting parameter itself automatically. Its stability has been proved by virtual simulation also. At present, SALEHD with this controller, has been put into practical application in multiple spacecraft types and achieved certain economic effect.

\section{References}

1. Tang Laiying, Sun Gang, Chen Jinming, Fu Hao. Design and research of Spacecraft Level-adjusted Hoist equipment based on method of suspension centre 
adjustment $[\mathrm{J}]$. Spacecraft environment engineering, 2013(2): 219-224

2. Fu Hao, Sun Gang, Tang Laiying, Cui Junfeng. Design and Development of Spacecraft Automatic Leveling and Equalizing Hoist Device. International Journal of mechanical aerospace industrial mechatronic and manufacturing engineering. Vol :6, No 9, 2012

3. LIU Yu-gang. The technology improvement of assembling scheme for hoisting tool of a satellite $[\mathrm{J}]$. Spacecraft environment engineering, 2004, 21(4): 48-53
4. ZHAO Pifu, QI Chun, CHEN Xianfeng. Study of hoisting technology of aerospace product $[\mathrm{J}]$. Aerospace manufacturing technology, 2007,(3): 12-16

5. Yi Yu, Jianqing Yi, Chengdong Li, Dongbin Zhao, Jianhong Zhang, Control of a Rope-driven Self-leveling Device for Leveling Adjustment, 2009 American Control Conference Hyatt Regency Riverfront, St.Louis, MO, USA. June 10 12,2009 